\title{
Aspects Épidémiologiques, Cliniques et Thérapeutiques des Tumeurs de la Parotide
}

\author{
Moussa M, \\ Service d'odonto-Stomatologie Hôpital National de Niamey, Niger \\ Abba Kaka H.Y, \\ Roufaye $L$, \\ Service d’ophtalmologie de l'Hopital National de Niamey, Niger \\ Eboungabeka Trigo ER, \\ Service de Stomatologie et Chirurgie Maxillo-Faciale du CHU de \\ Brazzaville, Congo \\ Bancole Pognon SA \\ Service d’odonto-Stomatologie CNHU HKM, Cotonou, Bénin
}

Doi:10.19044/esj.2020.v16n24p147 URL:http://dx.doi.org/10.19044/esj.2020.v16n24p147

\section{Résumé}

Introduction: Les pathologies des glandes salivaires sont relativement rares, dominées par les tumeurs de la glande parotide. Elles sont caractérisées par leur polymorphisme clinique et leur grande diversité morpho-histologique. Le traitement est surtout chirurgical, la complication majeure de cette chirurgie reste la paralysie faciale pouvant être transitoire ou permanente. Dans ce sens, l'objectif de ce travail était de décrire les aspects épidémiologiques, cliniques, paracliniques et thérapeutiques des tumeurs parotidiennes rencontrées dans le service de l'odontostomatologie de l'Hôpital National de Niamey (HNN) durant la période de l'étude. Méthodologie: Il s'agissait d'une étude rétrospective et descriptive portant sur les tumeurs parotidiennes rencontrées au service d'odontostomatologie de l'Hôpital National de Niamey au Niger. Par ailleurs, la tumeur était homogène dans 2 cas sur 4 et bénigne également une fois sur 2. Résultats: 12 patients ont été colligés sur une période de 29 mois. La prévalence hospitalière était de 0,11\% avec 4,97 cas par an. La tranche d'âge allait de 9 à 75 ans. Il y avait 8 hommes contre 4 femmes. La malignité a été suspectée cliniquement devant une masse dure, fixe, douloureuse avec présence d'adénopathies satellites et associée à une paralysie faciale. La parotidectomie totale conservatrice du nerf facial a été réalisée chez 10 patients sur 12 et la chimiothérapie a été préconisée chez 2 patients. L'examen ophtalmologique a été réalisé chez tous les patients à la recherche d'une atteinte oculaire ou de la glande lacrymale. L'histologie a été contributive dans tous les cas et les tumeurs bénignes dominaient dans 50\% 
des cas avec comme chef de fil l'adénome pléomorphe. Conclusion: Les tumeurs de la glande parotide sont rares et leur prise en charge thérapeutique est essentiellement chirurgicale. Leur évolution est en général favorable. Néanmoins, l'accent devra être mis sur la surveillance à long terme de certaines formes histologiques dont le risque de dégénérescence et de récidive est élevé.

Mots-clés: Glande Parotide, Adénome Pléomorphe, Parotidectomie

\title{
Epidemiological, Clinical, and Therapeutic Aspects of Parotid Tumors
}

\author{
Moussa M, \\ Service d'odonto-Stomatologie Hôpital National de Niamey, Niger \\ Abba Kaka H.Y, \\ Roufaye L, \\ Service d'ophtalmologie de l'Hopital National de Niamey, Niger \\ Eboungabeka Trigo ER, \\ Service de Stomatologie et Chirurgie Maxillo-Faciale du CHU de \\ Brazzaville, Congo \\ Bancole Pognon SA \\ Service d’odonto-Stomatologie CNHU HKM, Cotonou, Bénin
}

\begin{abstract}
Introduction: Pathologies of the salivary glands are relatively rare and are dominated by tumors of the parotid gland. They are characterized by their clinical polymorphism and their great morpho-histological diversity. The treatment is mainly surgical. The major complication of this surgery is facial paralysis which can be transient or permanent. The objective of this work is to describe the epidemiological, clinical, paraclinical, and therapeutic aspects of parotid tumors encountered in the odontostomatology department of Niamey National Hospital during the study period. Methodology: This study is a retrospective and descriptive study on parotid tumors encountered in the odontostomatology department of the Niamey National Hospital in Niger. In addition, the tumor was homogeneous in 2 out of 4 cases and also benign once in 2. Results: 12 patients were collected over a period of 29 months. The hospital prevalence was $0.11 \%$ with 4.97 cases per year. The age range was from 9 to 75 years old. There were 8 men against 4 women. The malignancy
\end{abstract}


was suspected clinically before a hard, fixed, and painful mass with the presence of satellite lymphadenopathy which is associated with facial paralysis. Total conservative facial nerve parotidectomy was performed in 10 out of 12 patients, and chemotherapy was recommended in 2 patients. The ophthalmic examination was performed in all patients looking for damage to the eyes or the lacrimal gland. Histology was contributory in all cases and benign tumors dominated in $50 \%$ of cases with pleomorphic adenoma as the lead. Conclusion: Tumors of the parotid gland are rare and their therapeutic management is essentially surgical. Their development is generally favorable. However, emphasis should be placed on the long-term monitoring of certain histological forms for which the risk of degeneration and recurrence is high.

Keywords: Parotid Gland, Pleomorphic Adenoma, Parotidectomi

\section{Introduction}

Les pathologies des glandes salivaires sont relativement rares, représentant 3 à $4 \%$ de l'ensemble des tumeurs de la face et du cou (Fassi et al., 2010; Lebeau, 2009). Ces affections sont dominées par les tumeurs des glandes salivaires principales (63\%) et principalement la parotide dans près de $90 \%$ des cas (Lebeau, 2009). Les tumeurs des glandes salivaires sont bénignes ou malignes, primitives ou secondaires développées aux dépens des éléments constitutifs de la glande. Ces tumeurs sont caractérisées par leur polymorphisme clinique et leur grande diversité morpho-histologique dominée par les tumeurs bénignes, notamment les adénomes pléomorphes (Fassi et al., 2010). Le principal motif de consultation est la tuméfaction faciale, notamment celle de la loge parotidienne. Le diagnostic repose sur l'examen clinique et les examens paracliniques comme l'histologie, l'échographie couplée ou non à la cytoponction et l'IRM. Le traitement demeure chirurgical en premier lieu, pouvant être associé à la radiothérapie externe (RTE) et/ou à la chimiothérapie en cas de lésion maligne. À cet égard, cette étude se propose de décrire les aspects épidémiologiques, cliniques, paracliniques et thérapeutiques des tumeurs parotidiennes rencontrées au service de d'odontostomatologie de l'Hôpital National de Niamey au Niger.

\section{Matériel d'étude}

$\mathrm{Au}$ cours de cette étude les dossiers des patients, le registre d'hospitalisation (entrées et sorties des patients), le registre des comptes rendus opératoires, le registre d'anesthésie réanimation, le registre des soins infirmiers et le registre du laboratoire d'anatomie pathologie ont été consultés. Les données recueillies ont été analysées par EPI INFO 2000 version 3.5.2 après réalisation d'un masque de saisie puis traitées par Microsoft Word 2010 et Excel. 


\section{Méthode d'étude}

Il s'agissait d'une étude rétrospective et descriptive portant sur 12 cas de tumeurs parotidiennes traitées dans le service de l'odontostomatologie de l'Hôpital National de Niamey sur une période de 29 mois allant du $1^{\text {er }}$ Janvier 2011 au 31 Mai 2013. Les variables étudiées ont été l’âge, le sexe et la fréquence des tumeurs de la parotide et leurs caractéristiques cliniques, paracliniques et thérapeutiques.

\section{Résultats}

Dans la présente étude, la fréquence des tumeurs parotidiennes était de 4,97 cas par an avec une prévalence hospitalière de $0,11 \%$. Les patients étaient d'âges compris entre 10 et 61 ans (extrêmes 9 et 75 ans). L'analyse de la Figure $\mathrm{N}^{0} 1$ montre une prédominance des patients de tranches d'âge de 21-30 ans et 4 cas sur 12 de 41-50 ans soit une proportion de 33,3\%. La tuméfaction faciale était le principal motif de consultation chez 9 patients.

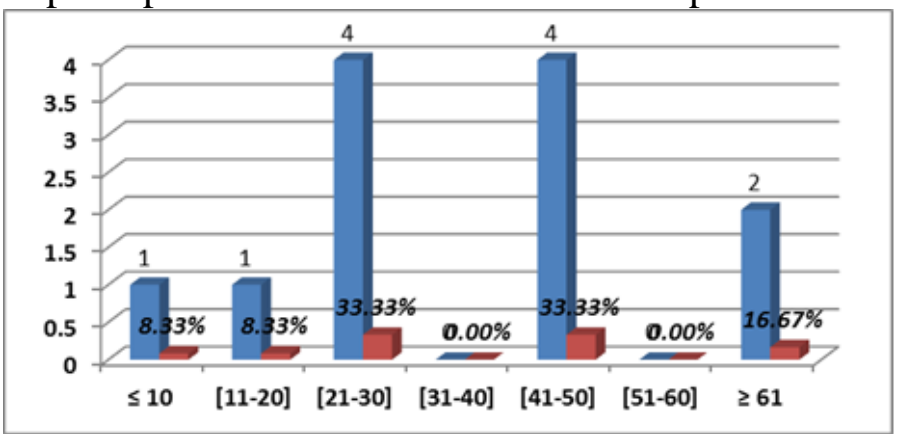

Figure 1. Répartition des patients selon la tranche d’âge

Dans la série de l'étude il a été relevé une prédominance masculine, 8 hommes (66,70\%) contre 4 femmes (33,30\%) soit un sex-ratio de 2 .

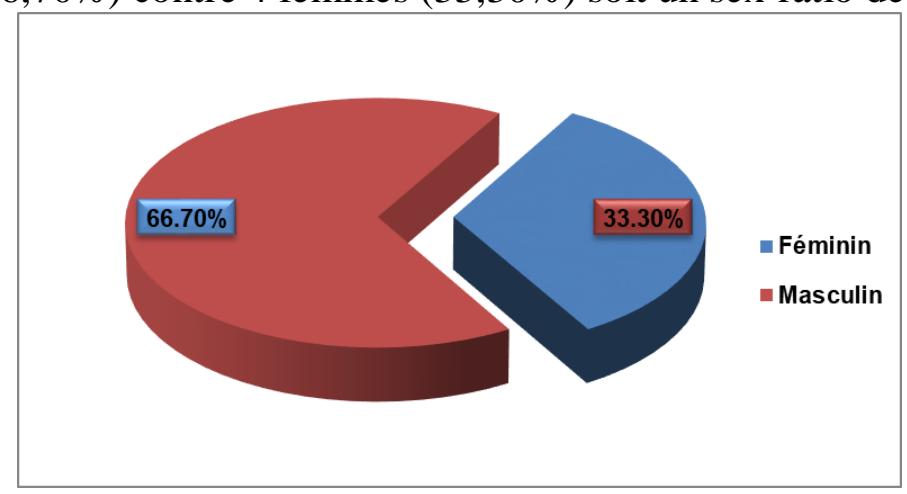

Figure 2. Répartition des patients selon le sexe 


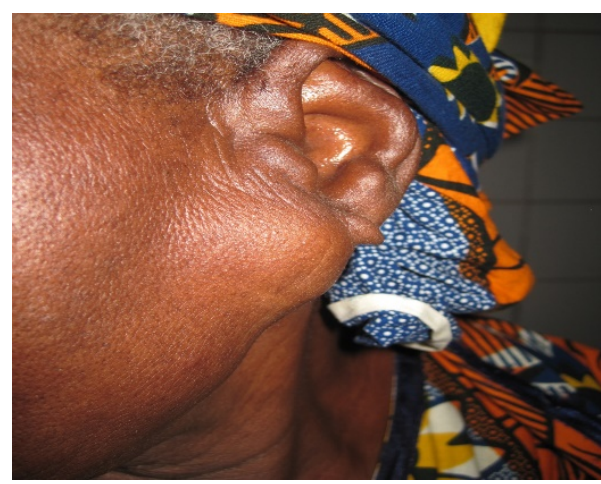

Figure 3. Tuméfaction en avant du tragus gauche refoulant le lobule de l'oreille (Source: Service de stomatologie de l'Hôpital National de Niamey/ Niger)

Le délai de consultation était de 2 à 36 mois. Dans la série étudiée huit(8) patients sur 12 avaient consulté plus de 12 mois après le début de la maladie. Le siège des tumeurs était à gauche dans 8 cas et à droite dans 4 cas. L'examen ophtalmologique a été systématique chez tous les patients ayant bénéficié de la parotidectomie; cet examen était sans particularité en dehors d'une paralysie palpébrale due à l'atteinte du nerf faciale chez certains patients. La répartition des sujets selon les caractéristiques de la tumeur est présentée dans le Tableau I.

Tableau I. Répartition selon les caractéristiques de la tumeur

\begin{tabular}{|c|c|c|}
\hline \multicolumn{2}{|c|}{ Caractéristiques } & Nombre de cas \\
\hline \multirow{2}{*}{ Coté atteint } & Droit & 4 \\
\cline { 2 - 3 } & Gauche & 8 \\
\hline Homogène & Oui & 11 \\
\hline Douloureuse & Oui & 3 \\
\hline Nerf facial & Atteint & 3 \\
\hline
\end{tabular}

L'échographie a été faite chez 4 patients. Elle a permis de connaître la localisation de la tumeur au lobe superficiel de la parotide chez 2 patients.

L'examen histologique avait retrouvé une fois sur 2 l'adénome pléomorphe. Les résultats des examens anatomo-pathologiques sont reportés dans le Tableau II.

Tableau II. Répartition selon la fréquence des tumeurs

\begin{tabular}{|c|c|}
\hline Formes histologiques & Nombre \\
\hline Adénome pléomorphe & 6 \\
\hline Carcinome adénoïde kystique & 2 \\
\hline Adénolymphome & 1 \\
\hline Carcinomes à cellules acineuses & 1 \\
\hline Lymphangiome & 1 \\
\hline Adénocarcinome polymorphe & 1 \\
\hline
\end{tabular}


Au plan thérapeutique, la chirurgie a été le principal moyen sauf pour les tumeurs malignes ou inopérables traitées par la chimiothérapie (2 cas). L’incision cutanée en S italique a été la voie d'abord la plus pratiquée (Figure 4).

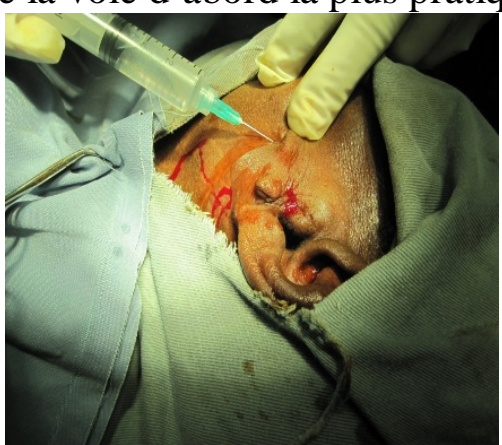

Figure 4. Infiltration à la xylocaine adrénalinée puis incision en $\mathrm{S}$ italique (Source: service d'odontostomatologie de l'Hôpital National de Niamey Niger)

La parotidectomie totale conservatrice du nerf facial a été pratiquée chez 10 patients (Figure 3 a et $b$ ).

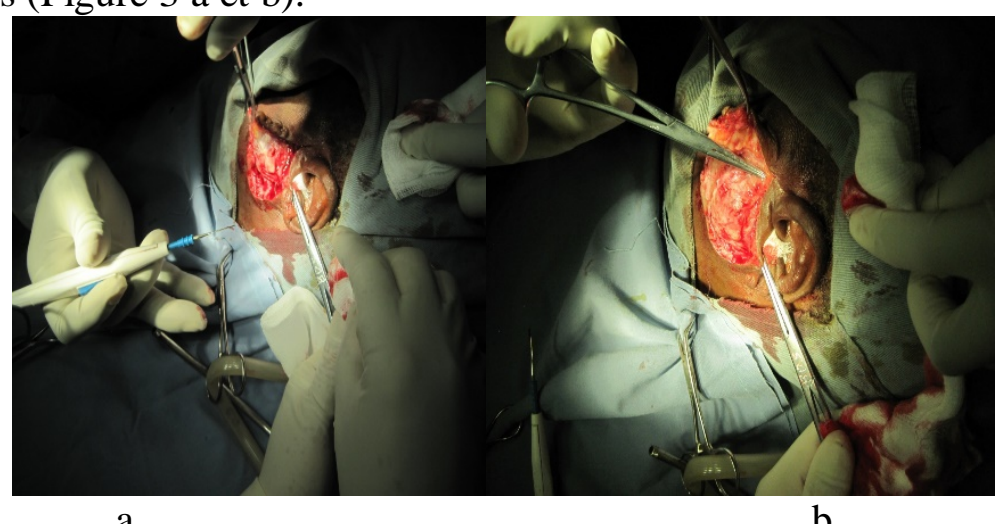

a

b

Figure 5. a et b Dissection du tissu parotidien avec isolement du nerf facial (Source: service d'odontostomatologie de l'Hôpital National de Niamey / Niger)

La masse tumorale a été totalement éliminée et les lambeaux suturés plan par plan.

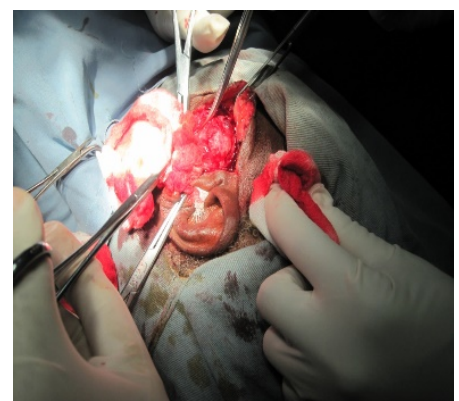

Figure 6. Exérèse de la masse tumorale

(Source: service d'odontostomatologie de l'Hôpital National de Niamey/Niger) 
Dans les 2 cas restants, les soins ont été palliatifs par chimiothérapie. Les suites opératoires étaient simples et l'évolution s’était faite vers la guérison chez 10 patients. La durée de l’hospitalisation allait de 0 à 14 jrs.

\section{Discussion}

Au plan épidémiologique: l'incidence globale des tumeurs parotidiennes dans la population est difficile à apprécier (Fassih et al., 2010). La prévalence hospitalière dans cette étude a été estimée à 0,11 des consultations faites au service de l'odontostomatologie de l'Hôpital National de Niamey avec une incidence moyenne d'environ 5 cas par an. La tuméfaction faciale était le principal motif de consultation chez 9 patients. Cette tuméfaction est située en général en avant du tragus refoulant le lobule de l’oreille (Figure 3). Dans une étude au Maroc, Essaadi et al. ont estimé la prévalence à 1,37\% dans une série de 120 cas en 9 ans, soit 13 cas/an, avec une incidence moyenne annuelle de 6 cas (Essaadi et al., 1998). De même, Séné au Sénégal avait rapporté une prévalence de 2,51\% pour une incidence moyenne annuelle de 11 cas dans une série de 218 cas colligés en 19 ans. Cette incidence faible retrouvée dans les séries est le signe de la rareté des tumeurs parotidiennes. La tranche d’âge de 21 à 30 ans était la plus touchée par cette tumeur dans l'intervalle de 9 à 75 ans investigué. Des résultats similaires ont été retrouvés par d'autres auteurs africains (Fassih et al., 2010; Essaadi et al., 1998; Diop et al., 1982). Il y avait une prédominance féminine dans la plupart des séries (Séné, 2001; Ouoba et al., 1998). Dans la série de cette étude par contre, il y avait 8 hommes contre 4 femmes. Cette prédominance masculine pourrait s’expliquer par le fait que les femmes n’aient pas le pouvoir décisionnel intimement lié à l’indépendance financière.

Le délai de consultation dans cette série allait de 2 mois à 36 mois. Des délais moyens de consultation plus longs allant de 36 mois à 77 mois avaient été rapportés dans différents travaux antérieurs dans la sous-région et au Maroc (Essaadi et al., 1998; Diop et al., 1982). Ces résultats montrent l'ampleur du retard à la consultation dans la région sujet de l'étude. Cet état de fait est d'autant plus accentué compte tenu du caractère indolore de la pathologie.

Au plan paraclinique: en l'absence d’orientation clinique évidente, la radio pulmonaire recherche des signes d'une tuberculose, des adénopathies médiastinales (lymphome), d'un cancer primitif, des métastases d'un cylindrome (Lebeau, 2009). Elle a été demandée chez tous les patients mais honorée 3 fois sur 4 .

La sialographie est l'examen de base des glandes salivaires principales (Ouoba et al., 1998). Elle permet d'étudier le parenchyme et le système canalaire (Lebeau, 2009). Mais elle ne suffit pas à elle seule pour poser le diagnostic de tumeurs parotidiennes (Ouoba et al., 1998). Certaines images sont évocatrices de malignité sans pouvoir l’affirmer (Lebeau, 2009). Selon 
Séné (2001) au Sénégal, la sialographie a un intérêt limité dans la pathologie tumorale, sa sensibilité pour le diagnostic des tumeurs étant seulement de $70 \%$. Dans la série de cette étude, aucune sialographie n’a été demandée du fait de l'extrême indigence des patients en général.

L'échographie, en plus d'être non invasive, peut indiquer la nature kystique de la lésion (Lebeau, 2009). Elle a été demandée chez tous les patients mais elle n’a été réalisée que par 4 patients sur 12, par manque de moyens financiers.

L'IRM est l'examen radiologique préférentiel devant une tumeur de la parotide car elle permet de préciser finement la morphologie, la taille et les rapports avec les tissus avoisinants (Fricain et al., 2017). Elle n’a pas été faite dans le cadre de ce travail en raison de sa non disponibilité au Niger.

La TDM est un examen permettant de bien apprécier les limites et l'extension d'un processus tumoral touchant les glandes salivaires vers les éléments du voisinage et les structures profondes, surtout quand on suspecte une extension basicrânienne (Lebeau, 2009). Elle est plus utile pour la planification chirurgicale que dans le diagnostic de la nature de la tumeur (Lebeau, 2009). Elle a été réalisée seulement chez 2 patients sur 12. Ce faible taux est dû au coût onéreux du scanner et à l'indigence de la quasi-totalité des sujets.

La certitude diagnostique est obtenue grâce à 3 examens que sont la cytoponction, la biopsie et la parotidectomie exploratrice. La cytoponction est un examen peu sensible et peu spécifique ; de plus, il est très opérateur dépendant (chirurgien et cytologiste). Elle a cependant une valeur d'orientation (Lebeau, 2009). Elle a été réalisée chez 2 patients sur les 12 de cette série. La biopsie à l'aveugle est fortement déconseillée car dangereuse pour le nerf facial et exposant au risque de dissémination tumorale et à la fistule salivaire (Lebeau, 2009). Elle n’a été faite pour aucun des patients de cette série. La parotidectomie exploratrice avec examen histologique est le seul examen de certitude pour le diagnostic étiologique d'une tuméfaction parotidienne (Lebeau, 2009). Elle a permis de retenir le diagnostic de l'adénome pléomorphe pour 6 patients. Nos résultats sont donc conformes aux données de la littérature, en effet, l’adénome pléomorphe est la plus fréquente des tumeurs parotidiennes, entre 50 à 70\% (Lebeau, 2009 ; Fricain et al., 2017) et Essaadi et al. au Maroc ont rapporté 49\% d'adénome pléomorphe dans leur série. Dans le cadre des tumeurs des glandes salivaires, plus la glande est petite, plus la lésion est susceptible d'être maligne. Ainsi, seulement $20 \%$ des tumeurs parotidiennes sont malignes (Fricain et al., 2017). Dans notre série, 4 cas de tumeurs sur 12 étaient malins.

Au plan thérapeutique: le geste chirurgical était la parotidectomie totale conservatrice du nerf facial (PTC) chez 10 patients. La PTC serait le type de chirurgie le plus réalisé par beaucoup de praticiens car elle minimise 
les risques de survenue de récidives tumorales et de lésions du nerf facial (Séné, 2001). De plus, l'argument du coût onéreux d'un traitement adjuvant comme la RTE (radiothérapie externe) devant éventuellement compléter une parotidectomie superficielle favoriserait le choix de la PTC. Les suites opératoires étaient simples dans les 10 cas opérés. Les complications éventuelles après la PTC seraient principalement la dépression rétromandibulaire, la fistule salivaire, la cicatrice prétragienne, le syndrome de Frey et les atteintes du nerf facial (Lebeau, 2009). Il est important d'en informer au préalable le patient. L'absence de visibilité de ces complications dans la série de cette étude pourrait s'expliquer par l'acceptation par les patients de quelques séquelles qu'ils considèreraient comme minimes par rapport aux bénéfices du traitement qu'ils avaient reçu. Spécifiquement, pour ce qui est de la dépression rétro-mandibulaire, parfois très inesthétique, le port de voile par les femmes et du turban par les hommes atténuerait sensiblement la gêne qu'elle occasionne. Dans cette étude aucune transformation maligne des tumeurs bénignes ni de récidive tumorale n’a été notée. Ceci serait certainement dû au court délai de surveillance des patients étudiés contrairement aux données de la littérature qui préconisent un suivi suffisant pour affirmer l'absence de récidive voire la guérison. Un recul de 10 ans serait nécessaire surtout pour les adénomes pléomorphes (Junien et al., 1979; Illé et al., 2016).

\section{Conclusion}

Les tumeurs de la glande parotide sont des lésions bénignes ou malignes, primitives développées aux dépens des éléments constitutifs de la glande; ou secondaires correspondant à un ensemencement tumoral de la parotide venu d'un autre organe. Elles sont caractérisées par leur grand polymorphisme clinique et histologique, sont de diagnostic relativement facile et sont en principe justifiables d'un traitement chirurgical associé, si besoin est, à une radiothérapie ou une chimiothérapie. L'évolution des tumeurs parotidiennes est généralement favorable après traitement. Néanmoins, l'accent devra être mis sur la surveillance à long terme des patients afin de déceler toute transformation cancéreuse et de prévenir d'éventuelles récidives. Conflits d'intérêt: Aucun conflit d'intérêt n’a été déclaré dans cette étude.

\section{References:}

1. Diop, EM., Dalo, KP., Ndiaye, PD., \& Diop, LS. (1982). Adénomes pléomorphes des glandes salivaires. Dakar Med, 27, 3, 371-83.

2. Essaadi, M., Jaji, A., Janah, A., Laraqui, NZ., Detsouli, M., \& Kadri (1998). Les tumeurs de la parotide (à propos de 120 cas). Revue Laryngol.Otol. Rhino, 119, 3, 177-82. 
3. Fassih, M., Lalya, I., Abada, A., Rouadi, S., \& Kadiri, F. (2010). Les tumeurs parotidiennes: étude épidémio-clinique et apport de l'imagerie dans le diagnostic. Rev marocaine du Cancer, 4 : 45-50.

4. Fricain, JC., Bodard, AG., \& Boisramé, S. (2017). Chirurgie orale ID Paris, 487-9.

5. Illé, S., Timi, N., Mahamane, MR., \& Kadre, OA. (2016). Les tumeurs de la glande parotide au Niger: Caractéristiques épidémiologiques , Cliniques et Histologiques. Health Sci.Dis: vol 17(3) July - August September , 63-66.

6. Junien Lavillauroy, C., Roux, O., \& Pasquier, R D. (1979). Les tumeurs de la parotide : étude anatomo'-c1inique, diagnostique et thérapeutique: à propos de 68 cas. Revue med. Alples Fr, 8, 1, 1-6.

7. Lebeau, J. (2009). Chirurgie maxilla-faciale et stomatologie pour le 2ème cycle des études médicales Masson Paris, p120.

8. Ouoba, K., Kabré, M., Sakandé, Dao M., \& Sanou, A. (1998). Résultats à court et moyen terme de la parotidectomie (à propos de 24 cas au CHU de Ouagadougou). Dakar Med , 143, 1, 114-7.

9. Séné, I. (2001). Etude des tumeurs de la parotide au service d'ORL du CHU Aristide Le Dantec. Thèse de Dakar, doctorat en médecine $\mathrm{N}^{\circ}$ 28, p101. 